\title{
SOME ASPECTS CONCERNING THE IMPACT OF THE END OF STROKE ON HYDRAULIC CYLINDERS
}

\author{
Niculai HAUK \\ "Dunarea de Jos" University of Galati, \\ nhauk@ugal.ro
}

\begin{abstract}
The increase of speeds for mechanical systems operated with hydraulic cylinder raises risks of mechanical impact of the end of the race. We are considering a number of measures to limit the impact intensity. Its size is estimated according to the masses in motion, to work pressures and to the geometry of the mechanism. Elasticity of the components is also an important variable. This paper presents a method which combines the classical calculation with numerical simulation systems.
\end{abstract}

\section{Keywords: hydraulic cylinder, cushioning, stress}

\section{Introduction}

In the technical applications use of the hydraulic cylinders is common. The use of hydraulic systems allows high energy densities in safe conditions.

Driven cinematic elements may be in rotation or translation. The moments of inertia and the masses of these elements are considerable and theirs working speeds are increasing.

Stopping motion of these elements is a difficult problem, especially when the hydraulic cylinder is moving to the end of the race. The stopping of movement by mechanical impact can be applied at low speed.

When the inertia is considerable, it is necessary to use hydraulic cushioning. This way of stopping requires a high pressure chamber. Braking may occur if:

- The hydraulic flow produced by pump that supplies the hydraulic cylinder is reduced;

- The pressure valve opens discharging a portion of the flow produced by the hydraulic pump;
- Both cases previously submitted are produced simultaneously.

The compressibility of hydraulic fluid also produces flow variations, but they are small. The hydraulic cushioning is made in diverse ways. The most commonly used ones are the systems with hydraulic resistance. These include bushings mounted on the piston-rod ensemble. They enter, at the end of stroke, precisely machined holes. This hydraulic resistance depends on the current position of the piston. Since the size of the hydraulic resistance is influenced by numerous factors (section, flow, viscosity, roughness) it is using an adjustable hydraulic resistance which works in parallel with that produced by cushioning sleeve. An example is shown in Figure 1.

The presence of the bushes creates an inconvenience by increasing the minimum length of the hydraulic cylinder to the same stroke. Decreases and the part of the race were that mobile assembly moves at full speed. 
The dimensions $\boldsymbol{a}$ and $\boldsymbol{b}$ (Figure 1) depend on the length of the damping bushes.

The dimensioning of the damping bushing is a problem at which the following are taken into consideration:

- Technological aspects related to maximum decelerations permits;

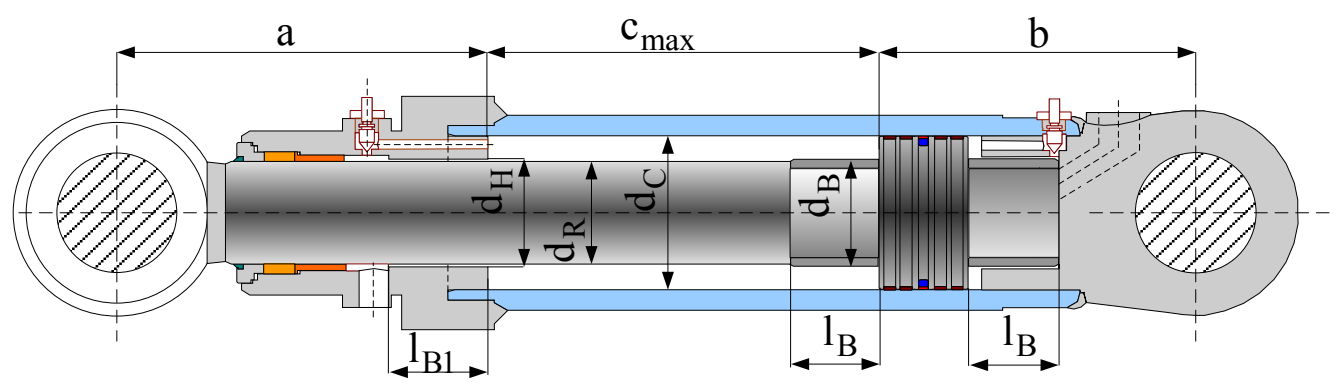

Figure 1: Dimensional structure of a hydraulic cylinder with cushioning system.

\section{Conditionalities and modes of analysis}

Stopping at end of stroke by a mechanical impact can lead to damage of the driven technological element or of the hydraulic cylinder.

In [1] is presented an analysis of the impact of the end of the stroke on the hydraulic cylinder. Based on the model presented we can make an estimate of stresses that occur when the hydraulic cylinder acts on the mass in translational motion.

Figure 2 shows a rigid element, 3, with fixed axis, acted by a hydraulic cylinder. In [2] the components of the motion law are presented:

- Angular positions
- Maximum permissible mechanical stresses, particularly in the hydraulic cylinder elements;

- The fulfilment of the kinematic and dynamic parameters by the driven machine. 


$$
\left\{\begin{array}{l}
\ddot{c}=f_{7}(\dot{c}, c, p) \\
\dot{p}_{1}=f_{8}(\dot{c}, c, p)
\end{array} .\right.
$$

From the integration of this system of differential equations results the functions that describe the functioning of the mechanism.

\section{Numerical simulations}

The effective solving of the system 4 is performed numerically, using the Runge Kutta method of order 4.

Figure 3 presents the results of numerical integration for the case of a hydraulic excavator arm. From all the numerical simulation results only those that are of interest to this paper have been kept. Their symbols correspond to the figures 1 and 2 . The parameter $\mathrm{Q}_{\mathrm{HC}}$ is the flow received by the hydraulic cylinder and $\mathrm{Q}_{\mathrm{S}}$ is the flow discharged through the safety valve.

The graphs in Figure 3 correspond to two numerical experiments. Differences arise from the use of damping bushings of different lengths. In the first case $1_{B}=100$ $\mathrm{mm}$ and in the second $\mathrm{l}_{\mathrm{B}}=40 \mathrm{~mm}$.

Although the movements start from the same initial position of the hydraulic cylinder and the stroke made is the same, the travel times are different. This is due to the use of shorter amortization bushes. The travel speed is high on a larger part of race for the second experiment. In the second experiment the space of braking decreases but the final speed of the mobile ensemble does not differ much from the first experiment.

The braking takes place in two phases:

- The first time the rate of delivery for the hydraulic cylinder decreases due to the flow produced by the hydraulic pump. The pump increases the pressure and reduces the flow because power is constant;

- It follows the phase where the active pressure exceeds the opening pressure of the pressure regulating valve and a part of the flow produced by the pump is drained at tank.

In the second experiment the energy losses are smaller.

With regard to cushioning pressures, $p_{2}$ important differences occur. Both the amplitude and the final pressure are higher in the second experiment. It is important to see the effects of these increases on the mechanical stress of the hydraulic cylinder body.

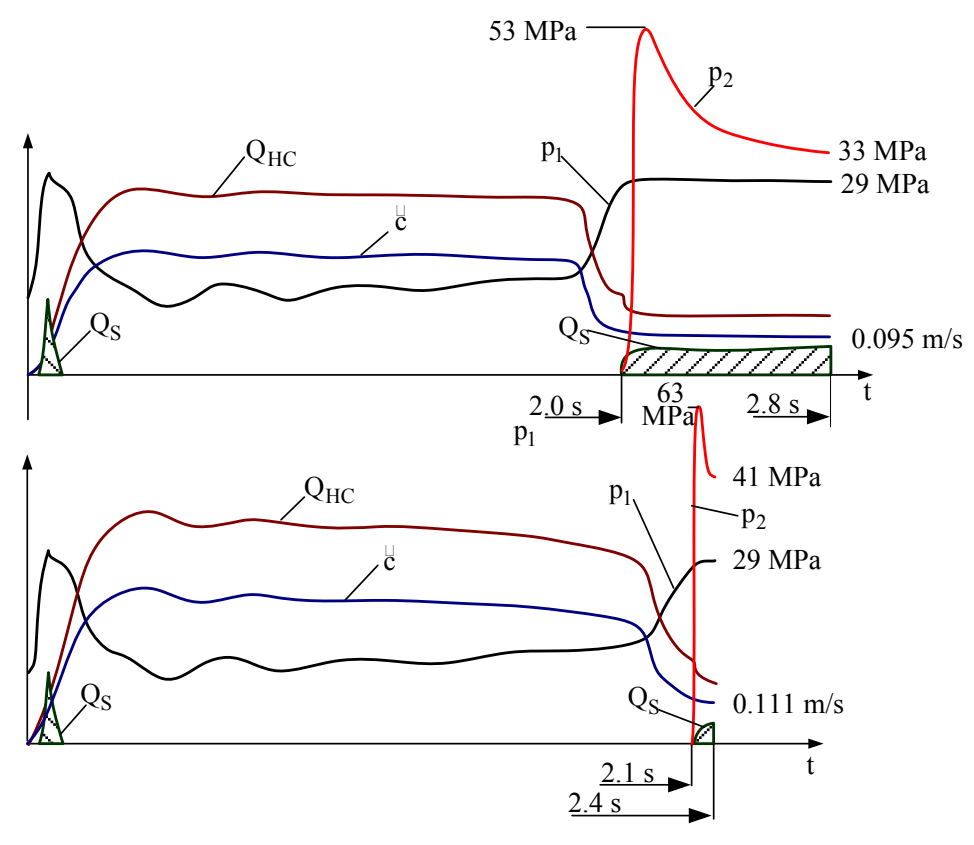

Figure 3: The effects of reducing the length of hydraulic damping bush on the functional parameters 


\section{Effects on the hydraulic cylinder}

In order to analyse the behaviour of the hydraulic cylinders, 3D drawings were made.

These models have been used to:

- Determine the parameters of flow regimes. Starting from 3D models fluid bodies were generated;

- Evaluate the consequences of reducing the length of the damping sleeve on the mechanical stresses occurring in the hydraulic cylinders.

The determinations were made by finite element method.

To calculate the mechanical stresses, the loads an surface were made based on the data provided by numerical simulations presented.

In the sealing area of the piston, it was considered an additional load. The location of this zone corresponds to the moment when the $p_{2}$ pressure reaches the maximum value. Figure 4 presents an example of such a loading.

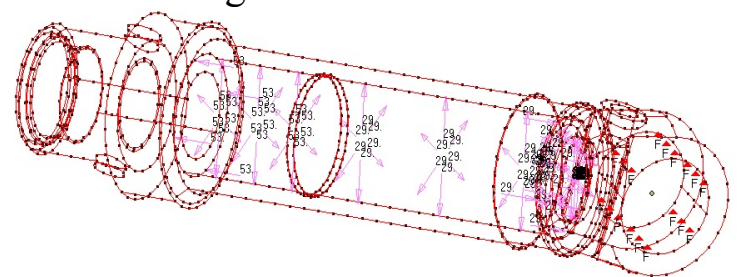

Figure 4: Uploads on hydraulic cylinder

With these elements was calculated the stress that occur in different parts of the body of the hydraulic cylinder, as can be seen from Figure 5.

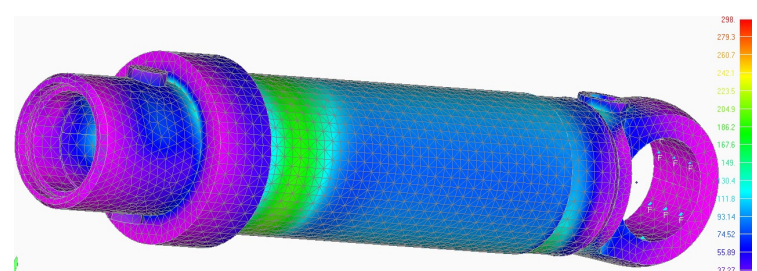

Figure 5: Stress in the hydraulic cylinder body

The maximum values were recorded for very short periods of time.

\section{Conclusions}

Choosing an inadequate length of damping sleeves can have unwanted effects. Their optimization is a process in several steps:

- Setting of the maximum speed permitting to avoid the mechanical damage;

- Determination of flow regimes;

- Selection and check of the length of damping sleeves, so that the final velocity is less than the maximum permitted speed;

- Check of maximal stresses in the cylinder parts occurring elements to be sure that they resist.

It should be noted that a number of other issues may influence behaviour of hydraulic cylinder. Mainly, it is hydraulic fluid temperature. The variation of viscosity with temperature is high and can significantly reduce braking capacity. Other factors with major influences are: the size of the clearance, the hydraulic fluid contamination with air, the kinematics of mechanism, etc. The method proposed provides the means for computing properly sized hydraulic cylinders.

\section{REFERENCES}

[1] Hauk N. Contributii teoretice si experimentale privind comportarea in regim dinamic a cilindrilor hidraulici montati pe excavatoare. Doctoral dissertation - University of "Dunarea de Jos" Galati, 1998.

[2] Hauk N. The influence of kinematics of mechanisms driven with hydraulic cylinders about the output of the system. Syrom01 Bucuresti 2001

[3] Janosevic D., Mitrev R., Andjelkovic B., Petrov .P, Quantitative measures for assessment of the hydraulic excavator digging efficiency, Journal of Zhejiang University-SCIENCE A (Applied Physics \& Engineering) 2012 13(12):926-942

[4] Poole A. D., Booker J. D. Design methodology and case studies in actuator selection. Mechanism and Machine Theory, Vol. 46. Issue 5 